\title{
The dynamics of sodium in sodium disilicate: Channel relaxation and sodium diffusion
}

\author{
Jürgen Horbach ${ }^{(1)}$, Walter $\operatorname{Kob}^{(2)}$, and Kurt Binder ${ }^{(1)}$ \\ (1) Institut für Physik, Johannes Gutenberg-Universität Mainz \\ Staudinger Weg 7, D-55099 Mainz, Germany \\ (2) Laboratoire des Verres - Université Montpellier 2 \\ Place E. Bataillon, cc 069, 34095 Montpellier, France
}

(October 27, 2018)

\begin{abstract}
We use molecular dynamics computer simulations to study the dynamics of amorphous $\left(\mathrm{Na}_{2} \mathrm{O}\right) 2\left(\mathrm{SiO}_{2}\right)$. We find that the $\mathrm{Na}$ ions move in channels em-

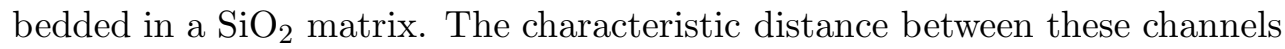
gives rise to a prepeak in the structure factor at around $q=0.95 \AA^{-1}$. The dynamics of sodium is given by a fast process which can be seen in the incoherent scattering function and a slow process which is seen in the coherent function. The relaxation time of the latter coincides with the $\alpha$-relaxation time of the matrix. The Kohlrausch exponent of the fast process for $q>1.6 \AA^{-1}$ is the same as the von Schweidler exponent for the slow one, demonstrating that the two processes are closely related.
\end{abstract}

PACS numbers: 61.20.Ja, 61.43.Fs, 66.30.Hs

Typeset using REVTEX 
The dynamics of mobile ions in glasses and melts is a field of great interest in geoscience, materials science, chemistry, and physics [1.2]. However, due to the lack of experiments that probe the dynamics on a microscopic level, many details of this dynamics are not understood well. A very valuable tool to understand these details are molecular dynamics (MD) computer simulations. Many of the recent MD studies of alkali silicate glasses [3] have given support to the idea of Ingram [4] that alkali ions move through "preferential pathways" and that these pathways, as has been suggested by Greaves [5], are related to a microsegregation of alkali ions. Very recently Jund et al. [6] investigated the dynamics of the Na ions in sodium tetrasilicate (NS4) and found no evidence for a clustering of sodium ions in individual configuration snapshots. However, these authors did identify preferential pathways in the dynamics of the sodium ions in that the sodium trajectories form a well connected network of pockets and connecting pathways. Moreover, they gave evidence for an uncorrelated motion of the sodium ions inside these channels, contrarily to the picture of a cooperative motion proposed by Greaves [5].

What so far has been left out from the discussion is the slow relaxation dynamics of the matrix, i.e. in sodium silicates the dynamics of the silicon and oxygen atoms. This is a very important issue since most of the models for the ion transport in glasses start from a coarse-grained representation of a glassy matrix which is not derived from first principles [2]. Thus the present paper is the first investigation in which the dynamics of the fast sodium ions is compared to the slow relaxation of the matrix and below we will show that these two processes are much closer related to each other than could naively be expected.

The system studied is $\left(\mathrm{Na}_{2} \mathrm{O}\right) 2\left(\mathrm{SiO}_{2}\right)$, NS2, i.e. a prototype of an ion conducting glass. The potential we use to describe the interactions between the ions in our MD simulation is a slight modification of the pair potential proposed by Kramer et al. which is based on $a b$ initio calculations [7]. More details on this potential can be found in Ref. [8]. The simulation was done at constant volume using 8064 particles $\left(N_{\mathrm{Si}}=1792, N_{\mathrm{Na}}=1792\right.$, and $\left.N_{\mathrm{O}}=4480\right)$ in a box of size $L=48.653 \AA$. This corresponds to a mass density of $2.37 \mathrm{~g} / \mathrm{cm}^{3}$, which is close to the experimental one [9]. The system was equilibrated at various temperatures in the range $2100 \mathrm{~K} \leq T \leq 4000 \mathrm{~K}$ before the production runs were started. These were done in the microcanonical ensemble, using the velocity form of the Verlet algorithm with a step size of $1.6 \mathrm{fs}$. At the lowest temperatures, the temperature for which the results presented here were obtained, these runs extended over $2.5 \mathrm{~ns}$ (1.5 million time steps) and in order to improve the statistics of the results we averaged over two independent runs.

In a recent publication we have shown that this model predicts structural and dynamical properties of NS2 which are in good agreement with experimental findings [8]. In particular we found that the static structure factor exhibits a prepeak at $q=0.95 \AA^{-1}$ which corresponds to a length scale of next-nearest $\mathrm{Na}-\mathrm{Na}$ or $\mathrm{Si}-\mathrm{Na}$ neighbors (around $6.6 \AA$ ). This result was very recently confirmed in a neutron scattering experiment by Meyer et al. [10] who found at this wave-vector a pronounced shoulder in the elastic intensities. Furthermore it has been shown that at temperatures $T \leq 2500 \mathrm{~K}$ the dynamics of the Na atoms is about two orders of magnitude faster than the one of silicon and oxygen atoms, which is in qualitative agreement with the experimental fact that this system is an ion conducting material [8]. Hence it is reasonable to assume that the present model does indeed give a good microscopic description of real NS2.

Using the same type of potential for NS4 it was recently shown that the Na atoms move 
in preferential pathways, also called "channels", which are basically frozen in structures inside the $\mathrm{SiO}_{2}$ matrix [6]. Furthermore it was found that the volume of these channels is relatively low and that they contain a large number of non-bridging oxygen atoms [11.12]. Hence it can be expected that the average density of the sodium atoms outside these channels is significantly lower than can be expected if the sodium atoms were distributed inside the system in a uniform way, i.e. that the diffusion of the sodium atoms is strongly restricted to a small subset of the configuration space. In order to check this idea we have calculated a (coarse grained) probability of finding no sodium atom at a given location in space. Following the approach of Jund et al. [6] we calculated this probability by dividing the system into $48^{3}$ cubes (of length $L / 48 \approx 1.01 \AA$ ). Then we calculate the probability $P(t)$ that a cube which does not contain a sodium ion at time zero is also not visited by a sodium ion until a later time $t$. The time dependence of $P(t)$ is shown in the inset of Fig. 1. From this graph we recognize that after $2.5 \mathrm{~ns}$, i.e. after more than the $\alpha$-relaxation time of the matrix [13, more than $50 \%$ of the cubes have not yet been visited by a sodium atom. (We mention that after this time the mean squared displacement of the Na atoms is more than $(45 \AA)^{2}$, which shows that these atoms have moved a large distance. On this time scale also the local structure of the $\mathrm{Si}-\mathrm{O}$ matrix is reconstructed [13].) Hence we can conclude that on this time scale the sodium free region forms a percolating structure around a network of channels, i.e. it has somewhat the structure of a Swiss cheese. In order to investigate the structure of this percolating region we define a "Swiss cheese" structure factor $S_{\mathrm{sc}}(q, t)$ as follows: We assign to each cube which has not been visited by a sodium atom until time $t$ a point and we compute the static structure factor of $N_{\mathrm{sc}}(t)=P(t)\left(48^{3}-N_{\mathrm{Na}}\right)$ points:

$$
S_{\mathrm{sc}}(q, t)=\frac{1}{N_{\mathrm{sc}}(t)} \sum_{k, l=1}^{N_{\mathrm{sc}}(t)} \exp \left(i \vec{q} \cdot\left(\vec{r}_{k}-\vec{r}_{l}\right)\right) .
$$

This quantity is shown in Fig. 1 1 for four different times: $t=0.56 \mathrm{ps}, 7.7 \mathrm{ps}, 164 \mathrm{ps}$, and $2.13 \mathrm{~ns}$. We see that $S_{\mathrm{sc}}(q, t)$ has peaks at $q_{1}=0.9 \AA^{-1}$ and $q_{2}=2.15 \AA^{-1}$ which are also the prominent features in $S_{\mathrm{NaNa}}(q)$, the static structure factor for the Na-Na correlations [8]. Hence we can now conclude that the peak at $q_{1}$ in $S_{\mathrm{NaNa}}(q)$, which has also been seen in nscattering experiments [10], corresponds to the typical distance between the channels. Note that with increasing time the height of this peak increases quickly. However, it is clear that the peak at $q_{1}$ decreases again on the time scale on which the matrix starts to reconstruct itself significantly and thus rearranges the channel structure.

The peak at $q_{2}$ reflects the length scale of nearest sodium neighbors [8]. As we see in Fig. [1 its height is constant for $7.7 \mathrm{ps} \leq t \leq 2.13 \mathrm{~ns}$. The fact that this peak is still present at $t=2.13$ ns means that there are preferred sites for the sodium ions inside the channels which is reasonable because most of the sodium ions are located in the neighborhood of dangling bonds [11.12] and these bonds do not disappear until the network regions have significantly rearranged themselves. The latter finding is also in agreement with the results from Ref. [6] for the distinct part of the van Hove correlation function.

We address now the question how the sodium ions relax inside the channels. An appropriate quantity to investigate this point are time dependent density-density correlation functions, i.e. the coherent intermediate scattering function $F(q, t)$ and its self part, the incoherent intermediate scattering function $F_{\mathrm{s}}(q, t)$ [14]. In Fig. 2 we show $F(q, t)$ for the 
$\mathrm{Na}-\mathrm{Na}$ correlations (solid lines) as well as $F_{\mathrm{s}}(q, t)$ for the sodium atoms (dashed lines) for three different wave-vectors: $q=0.94 \AA^{-1}, 2.0 \AA^{-1}$, and $3.5 \AA^{-1}$. From this figure we infer immediately a surprising result: At $q=0.94 \AA^{-1}$, i.e. at the characteristic $q$ value of the sodium channel structure, $F(q, t)$ decays on a time scale which is two orders of magnitude larger than the one for $F_{\mathrm{s}}(q, t)$. Such a strong difference cannot be explained by a de Gennes narrowing argument [14]. Instead this result can be rationalized by the idea that the sodium atoms move quickly between preferential sites, since this type of motion gives rise to a fast decorrelation of the incoherent function whereas it does not affect the coherent one. Only on the time scale of the relaxation of the $\mathrm{SiO}_{2}$ matrix also the coherent function starts to decay (see also Fig. 3, discussed below). Note that the slow decay of $F(q, t)$ is found only for wave-vectors around $0.95 \AA^{-1}$. For different $q$ the function decays significantly faster as can be seen from the other curves shown in Fig. 2 .

In order to quantify the typical relaxation time of the two mentioned processes we define the $\alpha$-relaxation times $\tau$ and $\tau_{\mathrm{s}}$ as the times at which $F(q, t)$ and $F_{s}(q, t)$, respectively, have decayed to 0.1. In Fig. 3 we show $\tau(q)$ for the $\mathrm{Si}-\mathrm{Si}$ and $\mathrm{O}-\mathrm{O}$ correlation, i.e. the relaxation time of the matrix for wave-vector $q$ (open symbols). Also included is $\tau(q)$ for the $\mathrm{Na}-\mathrm{Na}$ correlation and we see that for wave-vectors larger than $\approx 1.5 \AA^{-1}$ it is smaller than the characteristic relaxation time of the network. In contrast to this we find that for $q \lesssim q_{1}$ the relaxation time for $\mathrm{Na}-\mathrm{Na}$ is close to the one of the matrix. Moreover we see that $\tau(q)$ for the $\mathrm{Na}-\mathrm{Na}$ correlation is in phase with the corresponding partial structure factor $S_{\mathrm{Na}-\mathrm{Na}}(q)$ which is also shown in the figure. Furthermore we recognize from this figure that the relaxation time of all coherent correlators is largest at $q_{1}$, in agreement with the discussion of Fig. 2. Also included in the figure is $\tau_{s}$ for the sodium atoms and we see that it is indeed much smaller than the one of $F(q, t)$, which shows that there are indeed two different processes.

Having discussed the relaxation dynamics of the $\mathrm{SiO}_{2}$ matrix and the sodium atoms on a qualitative level, we proceed now to investigate this dynamics in more detail. In this context it is of interest that a calculation of Bosse and Kaneko within the mode coupling theory (MCT) [15] found that in a binary system of hard spheres with large size ratio the coherent and incoherent scattering functions decay on very different time scales [16]. Furthermore it was found that the diffusion constant of the small particles is orders of magnitude larger than the typical relaxation time of the larger particles. Thus qualitatively the behavior of that system was very similar to the present one, with the quasi frozen $\mathrm{SiO}_{2}$ matrix and the sodium atoms playing the role of the large and small particles, respectively. This similarity also suggests that MCT might be able to give a reliable qualitative description of the relaxation dynamics of the present system. Indeed we show elsewhere [13] that the incoherent and coherent dynamics of silicon and oxygen in NS2 can be nicely described by the universal predictions of MCT. In particular the time-temperature superposition principle for the $\alpha$-relaxation regime holds and the late $\beta$-relaxation regime, i.e. the time window in which a correlation function $\Phi_{q}(t)$ starts to fall below the plateau at intermediate times, can be described by a von Schweidler law,

$$
\Phi_{q}(t)=f_{q}^{\mathrm{c}}+h_{q} t^{b}
$$

where $f_{q}^{\mathrm{c}}$ is the height of the mentioned plateau in $\Phi_{q}(t)\left(=F(q, t)\right.$ or $\left.F_{\mathrm{s}}(q, t)\right)$, and $h_{q}$ is a prefactor. According to MCT the exponent $b$ in Eq. (2) should be a system universal 
constant, i.e. be independent of the correlator or $q$, and indeed all our time correlation functions can be fitted with Eq. (2) by fixing the exponent $b$ to 0.47 .

In the following we will relate this result with the relaxation dynamics of the sodium atoms as measured by $F_{\mathrm{s}}(q, t)$. The long time-decay of these functions can be well described by Kohlrausch laws,

$$
\Phi_{q}(t)=A_{q} \exp \left(-\left(t / \tau_{\mathrm{KWW}}(q)\right)^{\beta(q)}\right)
$$

The $q$-dependence of the exponent $\beta$ is shown in the inset of Fig. 1 . Very surprisingly we see that, for $q>1.6 \AA^{-1}, \beta$ seems to become independent of $q$ and is around $\beta_{\infty}=0.47$. That $\beta$ really becomes independent of $q$ is demonstrated in Fig. $⿴$ where we plot $F_{\mathrm{s}}(q, t) / A_{q}$ versus the rescaled time $t / \tau_{\mathrm{KWW}}$ for $1.7 \AA^{-1} \leq q \leq 4 \AA^{-1}$. (Note that $A_{q}$ and $\tau_{\mathrm{KWW}}$ have been obtained from fits to $F_{\mathrm{s}}(q, t)$ in which $\beta$ was fixed to $\beta_{\infty}$.) If $\beta=\beta_{\infty}$ really holds the curves for the different $q$ should fall onto a master curve in the $\alpha$-relaxation regime and they do so for $t / \tau_{\mathrm{KWW}}>0.1$. That this is a nontrivial result is demonstrated by the curve for $q=0.5$ which is included in the figure as well.

We note that the value of $\beta_{\infty}$ coincides with the value of the von Schweidler exponent $b$ from Eq. (2). This important observation can be rationalized by the prediction of Fuchs, obtained within a MCT calculation, that the Kohlrausch law becomes exact for $q \rightarrow \infty$ in which case $\beta_{\infty}$ is equal to the von Schweidler exponent $b$ [17]. Fuchs has argued that this result can be interpreted using Levy's generalization of the central limit theorem [18]: For large $q$ the $\alpha$-relaxation of the correlators is the result of a sum of many independent terms, each of which follows the von Schweidler law Eq. (2). According to Levy's central limit theorem this sum is the characteristic function of a stable distribution and the latter is exactly the Kohlrausch law with exponent $b$. For finite values of $q$ the short time asymptote of the $\alpha$-relaxation regime is still a von Schweidler law but one has strong correlations of correlators with different $q$. So one has no longer the sum of independent processes, and the Kohlrausch law with exponent $b$ is no longer a solution for the $\alpha$-relaxation regime. In this sense the theory of the $\alpha$-process in MCT is a generalization of Levy's central limit theorem.

If we apply this probabilistic interpretation of $F_{\mathrm{s}}(q, t)$ for sodium we obtain two important results: Firstly Levy's central limit theorem can already be applied for $q>1.6 \AA^{-1}$, i.e. deviations become important only on length scales which are larger than the nearest neighbor distance between sodium atoms. Secondly the $\alpha$-processes for the self-motion of sodium ions and the relaxation of sodium channels have - although appearing on completely different time scales — the same short-time behavior given by a von Schweidler law with exponent $b=0.47$. So it seems that the fast diffusion of sodium ions through the channels has its slow counterpart in the relaxation of the matrix, i.e. the rearrangement of the channels at long times.

In summary we have found that the sodium trajectories in NS2 form channels in the $\mathrm{SiO}_{2}$ network, in agreement with recent results for NS4 [6]. The characteristic distance between these channels is of the order of $6.6 \AA$, i.e. the distance between second nearest $\mathrm{Na}-\mathrm{Na}$ or $\mathrm{Si}-\mathrm{Na}$ neighbors, and is reflected in the static structure factor as a prepeak at $q=0.95 \AA^{-1}$. Structural relaxation in NS2 is dominated by two processes: the fast sodium diffusion through the channels and the rearrangement of these channels on the time scale of the relaxation of the $\mathrm{SiO}_{2}$ matrix. The von Schweidler exponent $b$ for the system can also be 
found in the incoherent intermediate scattering function for sodium in that for $q>1.6 \AA^{-1}$ its long time decay is given by a Kohlrausch law with $\beta_{\infty}=b$. This shows that the relaxation of channels at long times and the fast sodium diffusion inside these channels are intimately connected to each other.

Acknowledgments: Part of this work was supported by the DFG through SFB 261/D1. We thank the RUS for a generous grant in computer time. 


\section{REFERENCES}

[1] K. L. Ngai, J. Non-Cryst. Sol. 203, 232 (1996); M. D. Ingram, Phys. Chem. Glasses 28, 215 (1987); S.R. Elliott, Solid State Ionics 70/71, 27 (1994); S. Sen, A.M. George, and J.F. Stebbins, J. Non. Cryst. Solids 197, 53 (1996); K. Funke and C. Cramer, Curr. Opin. Solid State Mater. Sci. 2, 483 (1997); S. Sen and J.F. Stebbins, Phys. Rev. B 55, 3512 (1997).

[2] P. Maass, A. Bunde, and M.D. Ingram, Phys. Rev. Lett. 68, 3064 (1992).

[3] B. Vessal, M. Amini, P. T. Marten, A. V. Chadwick, C. R. A. Catlow, R. Mole, and S. Houde-Walter, Nature 356, 504 (1992); W. Smith, G. N. Greaves, and M. J. Gillan, J. Chem. Phys. 103, 3091 (1995); J. Oviedo and J. F. Sanz, Phys. Rev. B 58, 9047 (1998).

[4] M. D. Ingram, Phil. Mag. B 60, 729 (1989).

[5] G. N. Greaves, J. Non-Cryst. Sol. 71, 203 (1985).

[6] P. Jund, W. Kob, and R. Jullien, Phys. Rev. B 64, 134303 (2001).

[7] G. J. Kramer, A. J. M. de Man, and R. A. van Santen, J. Am. Chem. Soc. 64, 6435 (1991).

[8] J. Horbach, W. Kob, and K. Binder, Phil. Mag. B 79, 1981 (1999); Chem. Geol. 174, 87 (2001).

[9] O. V. Mazurin, M. V. Streltsina, and T. P. Shvaiko-Shvaikovskaya, Handbook of Glass Data, Part A: Silica Glass and Binary Silicate Glasses (Elsevier, Amsterdam, 1983).

[10] A. Meyer, H. Schober, and D. B. Dingwell, submitted to Phys. Rev. Lett.

[11] S. Ispas, M. Benoit, P. Jund, and R. Jullien, Phys. Rev. B, in press.

[12] E. Sunyer, P. Jund, W. Kob, and R. Jullien, preprint 2001.

[13] J. Horbach, W. Kob, and K. Binder, to be published.

[14] J. P. Boon and S. Yip, Molecular Hydrodynamics (Dover Publications, New York, 1980).

[15] For reviews of the theory and tests of MCT see e.g. L. Sjögren and W. Götze, Rep. Prog. Phys. 55, 241 (1992); W. Götze, J. Phys.: Condens. Matter 11, A1 (1999).

[16] J. Bosse and Y. Kaneko, Progr. Theor. Phys. Suppl. 126, 13 (1997).

[17] M. Fuchs, J. Non-Cryst. Sol. 172-174, 241 (1994).

[18] B. V. Gnedenko and A. N. Kolmogorov, Limit Distributions for Sums of Independent Random Variables (Addison Wesley, Reading Mass., 1954). 


\section{FIGURES}

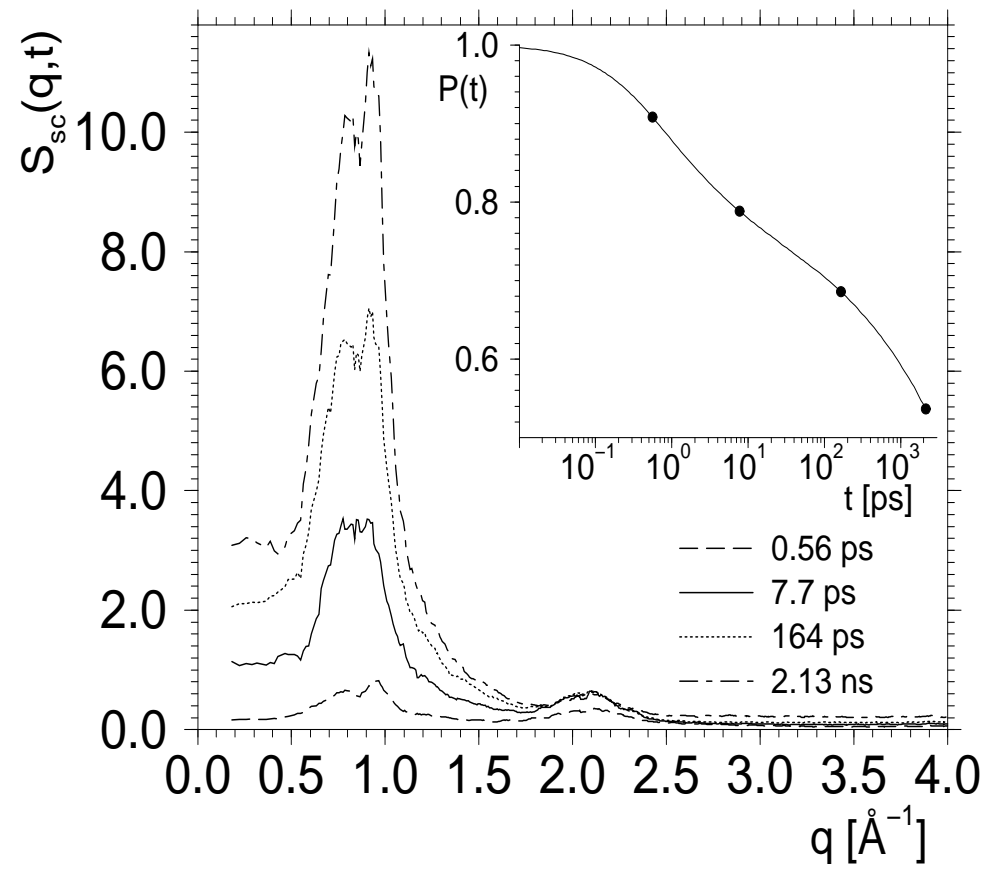

FIG. 1. "Swiss cheese" structure factor $S_{\mathrm{sc}}(q, t)$ for the sodium free regions at $T=2100 \mathrm{~K}$ for $t=0.56 \mathrm{ps}, 7.7 \mathrm{ps}, 164 \mathrm{ps}$, and $2.13 \mathrm{~ns}$. The inset shows the probability $P(t)$ that a cube which is sodium free at time zero remains sodium free until time $t$. The circles on the curve for $P(t)$ are at the times at which $S_{\mathrm{sc}}(q, t)$ is shown.

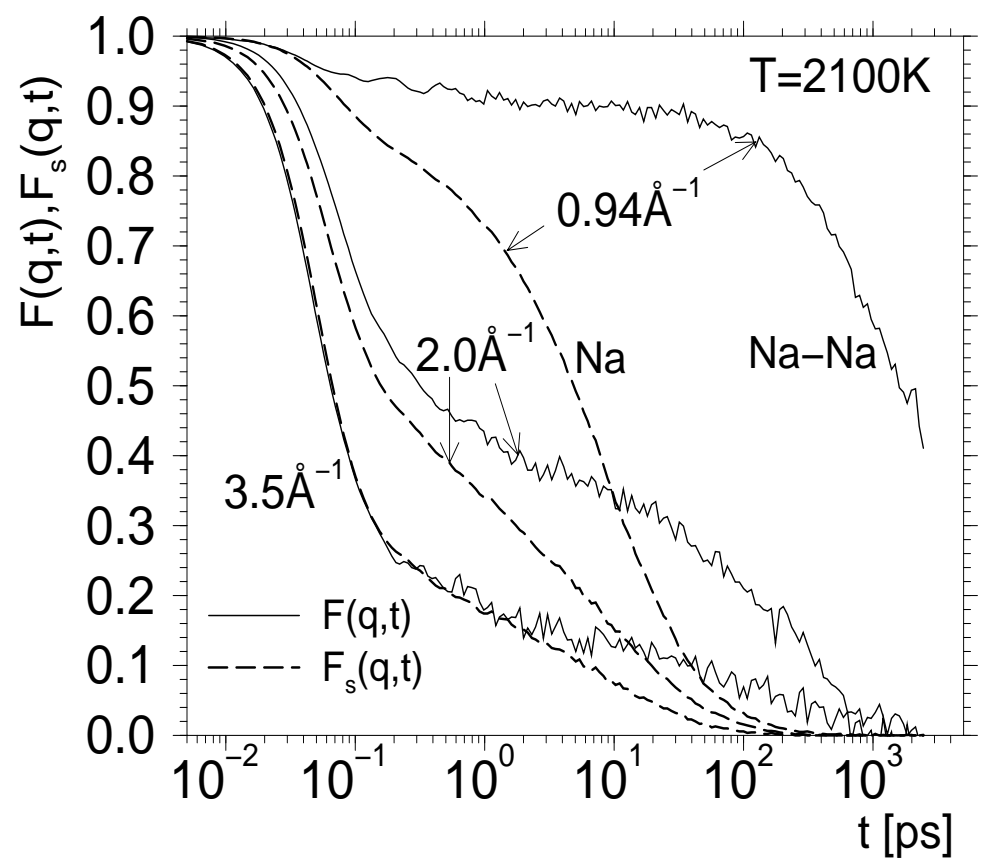

FIG. 2. Coherent intermediate scattering functions $F(q, t)$ for the sodium-sodium correlations (solid lines) and incoherent intermediate scattering functions $F_{\mathrm{s}}(q, t)$ (dashed lines) at $T=2100 \mathrm{~K}$ for the indicated values of $q$. 


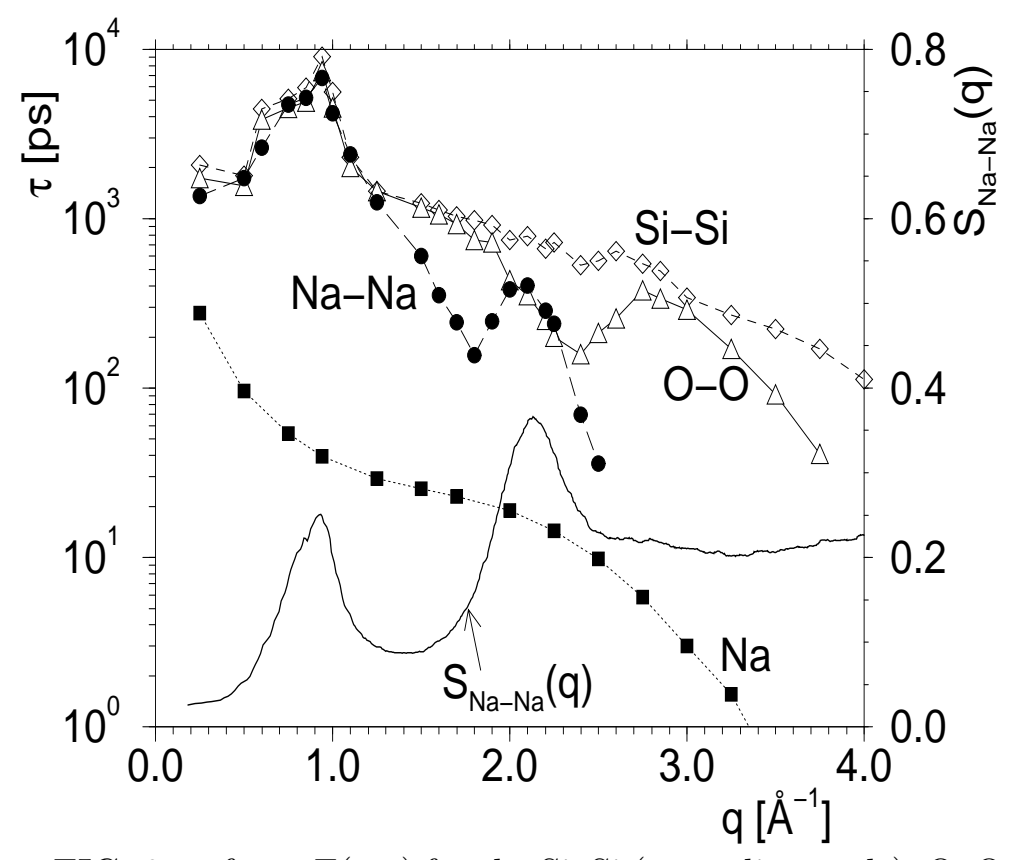

FIG. 3. $\tau$ from $F(q, t)$ for the $\mathrm{Si}-\mathrm{Si}$ (open diamonds), $\mathrm{O}-\mathrm{O}$ (triangles), and $\mathrm{Na}-\mathrm{Na}$ (filled circles) correlations and $\tau_{\mathrm{s}}$ from $F_{\mathrm{s}}(q, t)$ for $\mathrm{Na}$ (filled squares). The labels for the $y$ axis on the right-hand side are for the partial static structure factor $S_{\mathrm{Na}-\mathrm{Na}}(q)$ (bold solid line).

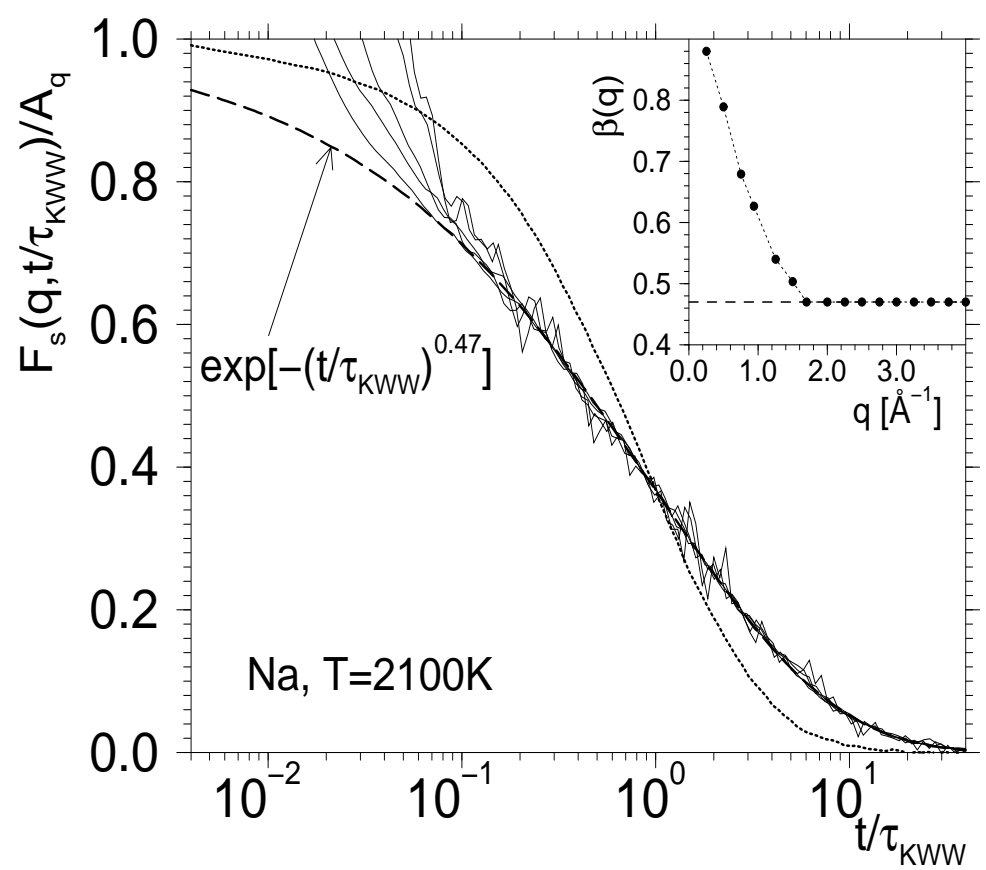

FIG. 4. The solid lines show $F_{\mathrm{s}}\left(q, t / \tau_{\mathrm{KWW}}(q)\right) / A_{q}$ for $q=1.7 \AA^{-1}, 2.0 \AA^{-1}, 2.75 \AA^{-1}, 3.5 \AA^{-1}$, and $4.0 \AA^{-1}$ (from left to right). (See text for the determination of $A_{q}$ and $\tau_{\mathrm{KWW}}$.) The dotted line shows the same quantity for $q=0.5 \AA^{-1}$. The dashed line is the Kohlrausch law $\Phi(\hat{t})=\exp \left(-\left(t / \tau_{\mathrm{KWW}}\right)^{0.47}\right)$. The inset shows the Kohlrausch exponent $\beta$ as a function of $q$. 